\title{
On the UV Spectra of the Side Chain of Copolypeptide in $\alpha$-Helical Conformation
}

\author{
Akinori KIDERa, Toshio Hayashi, and Akio Nakajima \\ Department of Polymer Chemistry, Kyoto University \\ Sakyo-ku, Kyoto 606, Japan.
}

(Received June 8, 1978)

\begin{abstract}
KEY WORDS Ultraviolet Spectra / Molar Absorptivity / Benzyl
Ester / Copolypeptide / $\gamma$-Benzyl L-Glutamate / L-Valine / L-Alanine /
\end{abstract}

Generally speaking, the chromophore in a polymer side chain follow the Lambert-Beer's law. ${ }^{1}$ However it has been found that the copolymer composition of the copolypeptide, containing $\gamma$-benzyl L-glutamate (BLG) as one of the components, affects the molar absorptivity of the benzyl side chain. In this paper, our observation on this phenomenon is discussed.

\section{EXPERIMENTAL}

\section{Materials}

Poly $(\gamma$-benzyl L-glutamate) (PBLG), copoly $(\gamma$ benzyl L-glutamate/L-valine) (GV) and copoly( $\gamma$-benzyl L-glutamate/L-alanine) (GA) were synthesized by the NCA method. Polymerization was carried out at room temperature in a $1: 1(\mathrm{v} / \mathrm{v})$ mixture of dioxane and methylene dichloride in the presence of triethylamine as an initiator. Table I gives the intrinsic viscosity, [ $\eta$ ], in dichlo-

Table I. Characterization of polymers

\begin{tabular}{ccc}
\hline Sample & $\begin{array}{c}{[\eta](\mathrm{d} l / \mathrm{g})} \\
\left(\mathrm{DCA}, 25^{\circ} \mathrm{C}\right)\end{array}$ & $\begin{array}{c}\text { BLG content } \\
(\mathrm{mol} \%)\end{array}$ \\
\hline GV-1 & 1.55 & 98.7 \\
$\mathrm{GV}-2$ & 1.75 & 96.7 \\
$\mathrm{GV}-3$ & 1.41 & 94.5 \\
GA-1 & 1.47 & 92.8 \\
GA-2 & 1.51 & 85.1 \\
PBLG & 1.42 & 100.0 \\
\hline
\end{tabular}

roacetic acid and the composition of the copolypeptide determined by amino acid analysis. The amino acid analyses were carried out at the Protein Research Foundation, Osaka.

\section{Preparation of Solution}

Mixtures of dioxane (DO) and trifluoroacetic acid (TFA) were used as solvent. Each of the mixtures dissolves completely the copolypeptides in $\alpha$-helical conformation, and the benzyl chromophore in the ultraviolet (UV) region could be measured without any interference by the solvent absorption. It was also confirmed that the benzyl ester group in the side chain of PBLG was not debenzylated in these solvent systems. DO was purified just before use by a standard procedure. TFA was purchased from the Protein Research Foundation and used without further purification.

\section{Absorption Measurements}

The absorption of benzyl ester group in the UV region was measured at room temperature at wavelength ranging from $255-270 \mathrm{~nm}$, using the HITACHI EPS-3T recording spectrophotometer; it was difficult to measure quantitatively the absorbance below $255 \mathrm{~nm}$ owing to TFA absorption. The measurements were carried out at three to five different concentrations, and the absorbance of the maximum at $259 \mathrm{~nm}$ was plotted against the concentration; the maximum at $259 \mathrm{~nm}$ is the largest one in the benzyl ester chromophore in the UV region. ${ }^{2}$ The molar absorptivity was calculated from the gradient of the plot after confirming that it was linear and its intercept was zero.

\section{RESULTS AND DISCUSSION}

Figure 1 shows the relation between the molar absorptivity and the BLG content ( $\mathrm{mol} \%$ ) in the copolypeptide. The experimental points are re- 


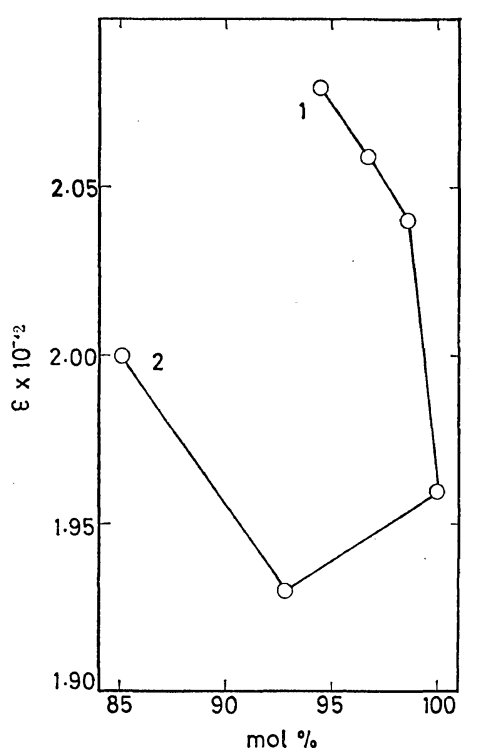

Figure 1. Dependence of molar absorptivity on BLG content in copolypeptides: (1) GV and (2) GA.

Table II. BLG Content measured by means of two methods (mol \%)

\begin{tabular}{lcc}
\hline \hline Sample & UV method $^{\mathrm{a}}$ & $\mathrm{AAA}^{\mathrm{b}}$ \\
\hline GV-1 & 108 & 98.7 \\
GV-2 & 108 & 96.7 \\
GV-3 & 108 & 94.5 \\
GA-1 & 88.8 & 92.8 \\
GA-2 & 90.1 & 85.1 \\
\hline
\end{tabular}

a On the basis of the molar absorptivity $(=196)$ of PBLG.

b Amino acid analysis, the same as shown in Table I.

producible within $\pm 0.3 \%$ for the abscissa and $\pm 0.02 \times 10^{2}$ units for the ordinate. As is obvious from Figure 1, the presence of valyl or alanyl residue in the GV or GA copolymer varies the molar absorptivity of the benzyl ester group of the BLG residue. Thus, if the compositions of these copolypeptides are determined only by the absorbance at $259 \mathrm{~nm}$, a considerable degree of errors will be expected as is obvious in Table II.

It is generally known that the van der Waals interaction causes an absorption shift of the UV spectrum and transforms its vibrational structure. $^{2,3}$ Actually, the vibrational structure of benzyl ester group was deformed by increasing the TFA vol $\%$ in the solvent mixture as shown in

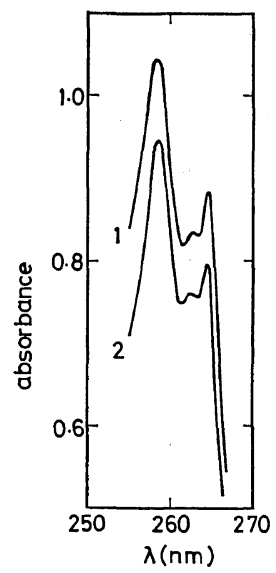

(a)

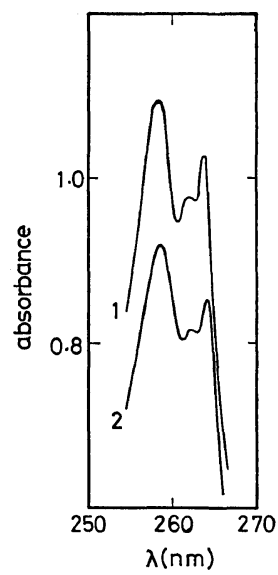

(b)
Figure 2. Ultraviolet spectra of PBLG and benzyl acetate. (a) In dioxane-TFA (9:1): 1, benzyl acetate (concn: $5.21 \mathrm{mmol} / \mathrm{l}$ ); 2, PBLG (concn: 4.82 $\mathrm{mmol} / l)$. (b) In dioxane-TFA $(8: 2): 1$, benzyl acetate (concn: $7.58 \mathrm{mmol} / l$ ); 2 , PBLG (concn: $5.56 \mathrm{mmol} / l$ ).

Figure 2. However, the frequency dependence, i.e., the positions and intensities of maxima, were almost identical for all the copolypeptides in the same solvent system. Further, there was a great similarity in the spectra of PBLG and benzyl acetate, as shown in Figure 2. This shows that the environment in the vicinity of benzyl ester group which may influence the electrons of benzyl ester, is not affected by the situation in which the chromophore is on the side chain of the $\alpha$-helical copolypeptide.

From the above discussion, it is concluded that the result shown in Figure 1 should be attributed to causes other than the electronic interaction of the chromophore. Firstly, it can be assumed that the absorbance is altered by varying the electric field surrounding benzyl ester group, which depends on the dielectric constant of the solvent system in the vicinity of benzyl ester group. It is usually observed that the solubility of copolypeptide is varried with its composition to a great extent. Therefore, it seems that the dielectric constant of the solvent system may be altered locally by changing the copolymer composition, even though it does not affect directly the electronic state of the chromophore. ${ }^{4}$ Secondarily, concerning the solubility, it can be also assumed that the degree of hypochromic effect of benzyl ester group is 
altered by the side chain conformation, since the side chain conformation of polypeptide varies with the kind of helicogenic solvent, ${ }^{5}$ and the degree of hypochromic effect of the side chain depends on its conformation. ${ }^{6}$ In this case, it is expected that the side chain conformation of BLG is affected by the composition of comonomers in copolypeptide chains.

\section{REFERENCES}

1. R.E. Nylund and W.G. Miller, J. Am. Chem. Soc., 87, 3537 (1965).

2. D.B. Wetlaufer, Adv. Protein Chem., 17, 345(1962).

3. N. Mataga and T. Kubota, "Molecular Interaction and Electronic Spectra," M. Dekker, New York, N. Y., 1970, Chapter 8.

4. J. M. G. Cowie, J. Polym. Sci., C23, 267 (1968).

5. Y. Ishimuro, F. Hamada, and A. Nakajima, Polym. Prepr., Jpn., 25, 1241 (1976).

6. M. T. Vala, Jr. and S. A. Rice, J. Chem. Phys., 39, 2348 (1963). 